\title{
Determination of the Pole Position of the Lightest Hybrid Meson Candidate
}

\author{
A. Rodas, ${ }^{1, *}$ A. Pilloni,${ }^{2,3, \dagger}$ M. Albaladejo, ${ }^{2,4}$ C. Fernández-Ramírez, ${ }^{5}$ A. Jackura, ${ }^{6,7}$ V. Mathieu, ${ }^{2}$ \\ M. Mikhasenko, ${ }^{8}$ J. Nys, ${ }^{9}$ V. Pauk, ${ }^{10}$ B. Ketzer, ${ }^{8}$ and A. P. Szczepaniak ${ }^{2,6,7}$
}

(Joint Physics Analysis Center)

\author{
${ }^{1}$ Departamento de Física Teórica, Universidad Complutense de Madrid, E-28040 Madrid, Spain \\ ${ }^{2}$ Theory Center, Thomas Jefferson National Accelerator Facility, Newport News, VA 23606, USA \\ ${ }^{3}$ European Centre for Theoretical Studies in Nuclear Physics and Related Areas (ECT*) and Fondazione Bruno Kessler, \\ I-38123 Villazzano (TN), Italy \\ ${ }^{4}$ Departamento de Física, Universidad de Murcia, E-30071 Murcia, Spain \\ ${ }^{5}$ Instituto de Ciencias Nucleares, Universidad Nacional Autónoma de México, Ciudad de México 04510, Mexico \\ ${ }^{6}$ Center for Exploration of Energy and Matter, Indiana University, Bloomington, IN 47403, USA \\ ${ }^{7}$ Physics Department, Indiana University, Bloomington, IN 47405, USA \\ ${ }^{8}$ Universität Bonn, Helmholtz-Institut für Strahlen- und Kernphysik, 53115 Bonn, Germany \\ ${ }^{9}$ Department of Physics and Astronomy, Ghent University, Ghent 9000, Belgium \\ ${ }^{10}$ Institut für Kernphysik \& PRISMA Cluster of Excellence, Johannes Gutenberg Universität, D-55099 Mainz, Germany
}

(Received 13 October 2018; revised manuscript received 19 November 2018; published 29 January 2019)

\begin{abstract}
Mapping states with explicit gluonic degrees of freedom in the light sector is a challenge, and has led to controversies in the past. In particular, the experiments have reported two different hybrid candidates with spin-exotic signature, $\pi_{1}(1400)$ and $\pi_{1}(1600)$, which couple separately to $\eta \pi$ and $\eta^{\prime} \pi$. This picture is not compatible with recent Lattice QCD estimates for hybrid states, nor with most phenomenological models. We consider the recent partial wave analysis of the $\eta^{(\prime)} \pi$ system by the COMPASS Collaboration. We fit the extracted intensities and phases with a coupled-channel amplitude that enforces the unitarity and analyticity of the $S$ matrix. We provide a robust extraction of a single exotic $\pi_{1}$ resonant pole, with mass and width $1564 \pm 24 \pm 86$ and $492 \pm 54 \pm 102 \mathrm{MeV}$, which couples to both $\eta^{(\prime)} \pi$ channels. We find no evidence for a second exotic state. We also provide the resonance parameters of the $a_{2}(1320)$ and $a_{2}^{\prime}(1700)$.
\end{abstract}

DOI: $10.1103 /$ PhysRevLett.122.042002

Introduction.-Explaining the structure of hadrons in terms of quarks and gluons, the fundamental building blocks of quantum chromodynamics (QCD), is of key importance to our understanding of strong interactions. The vast majority of observed mesons can be classified as $q \bar{q}$ bound states, although QCD should have, in principle, a much richer spectrum. Indeed, several experiments have reported resonance candidates that do not fit the valence quark model template $[1,2]$, mainly in the heavy sector [3-7]. These new experimental results, together with rapid advances in lattice gauge computations, open new fronts in studies of the fundamental aspects of QCD, such as quark confinement and mass generation. Since gluons are the mediators of the strong interaction, QCD dynamics cannot

Published by the American Physical Society under the terms of the Creative Commons Attribution 4.0 International license. Further distribution of this work must maintain attribution to the author(s) and the published article's title, journal citation, and DOI. Funded by SCOAP . be fully understood without addressing the role of gluons in binding hadrons. The existence of states with explicit excitations of the gluon field, commonly referred to as hybrids, was postulated a long time ago [8-12], and has recently been supported by lattice [13-15] and phenomenological QCD studies [16-19]. In particular, a state with exotic quantum numbers $J^{P C}\left(I^{G}\right)=1^{-+}\left(1^{-}\right)$in the mass range $1.7-1.9 \mathrm{GeV}$ is generally expected. The experimental determination of hybrid hadron properties-e.g., their masses, widths, and decay patterns-provides a unique opportunity for a systematic study of low-energy gluon dynamics. This has motivated the COMPASS spectroscopy program [20,21] and the $12 \mathrm{GeV}$ upgrade of Jefferson Lab, with experiments dedicated to hybrid photoproduction at CLAS12 and GlueX [22,23].

The hunt for hybrid mesons is challenging, since the spectrum of particles produced in high energy collisions is dominated by nonexotic resonances. The extraction of exotic signatures requires sophisticated partial-wave amplitude analyses. In the past, inadequate model assumptions and limited statistics resulted in debatable results. The first 
reported hybrid candidate was the $\pi_{1}(1400)$ in the $\eta \pi$ final state [24-28]. Another state, the $\pi_{1}(1600)$, was later claimed in the $\rho \pi$ and $\eta^{\prime} \pi$ channels, with different resonance parameters [29,30]. The COMPASS experiment confirmed a peak in $\rho \pi$ and $\eta^{\prime} \pi$ at around $1.6 \mathrm{GeV}$ $[31,32]$ and an additional structure in $\eta \pi$, at approximately $1.4 \mathrm{GeV}$ [33]. A theoretical approach based on a unitarized $U(1)$-extended chiral Lagrangian predicted a $\pi_{1}$ state with mass of about $1400 \mathrm{MeV}$ decaying mostly into $\eta^{\prime} \pi$ [34]. A phenomenological unitary coupled-channel analysis of the $\eta^{(\prime)} \pi$ system from E852 data was instead not conclusive [35]. While the $\pi_{1}(1600)$ is close to the expectation for a hybrid, the observation of two nearby $1^{-+}$hybrids below $2 \mathrm{GeV}$ is surprising. This makes the microscopic interpretation of the $\pi_{1}(1400)$ problematic. Moreover, in the $S U(3)$ limit, Bose symmetry prevents the decay of a hybrid into $\eta \pi$ [36]. A tetraquark interpretation of the lighter state might be viable, and would explain why this state has eluded predictions in constituent gluon models. However, this interpretation would lead to the prediction of unobserved doubly charged and doubly strange mesons [37], and is unfavored in the diquark-antidiquark model $[38,39]$. Establishing whether there exists one or two exotic states in this mass region is thus a stringent test for the available phenomenological frameworks in the nonperturbative regime.

In Ref. [40] we analyzed the spectrum of the $\eta \pi D$-wave extracted from the COMPASS data. In this Letter, we extend the mass dependent study to the exotic $P$ wave, and present results of the first coupled-channel analysis of the $\eta^{(\prime)} \pi$ COMPASS data. We establish that a single exotic $\pi_{1}$ is needed and provide a detailed analysis of its properties. We also determine the resonance parameters of the nonexotic $a_{2}(1320)$ and $a_{2}^{\prime}(1700)$.

Description of the data.-We use the mass independent analysis by COMPASS of $\pi p \rightarrow \eta^{(\prime)} \pi p$, with a $190 \mathrm{GeV}$ pion beam [33]. We focus on the $P$ - and $D$-wave intensities and their relative phase, in both channels. The published data are integrated over the range of transferred momentum squared $-t_{1} \in[0.1,1] \mathrm{GeV}^{2}$. However, given the diffractive nature of the reaction, most of the events are produced in the forward direction, near the lower limit in $-t_{1}$. The $\eta^{(\prime)} \pi$ partial-wave intensities and phase differences are given in $40 \mathrm{MeV}$ mass bins, from threshold up to $3 \mathrm{GeV}$. Intensities are normalized to the number of observed events corrected by the detector acceptance. The errors quoted are statistical only; systematic uncertainties or correlations in the extraction of the partial waves were found to be negligible [41]. We thus assume that all data points are independent and normally distributed. As seen in Figs. 4(a) and 5(a) of Ref. [33], at the $\eta^{\prime} \pi$ mass of $2.04 \mathrm{GeV}$ there is a sharp falloff in the $P$-wave intensity, and a sudden change by $50^{\circ}$ in the phase difference between the $P$ and $D$ wave. This behavior might be attributed to another state. The E852 experiment claimed indeed a third exotic $\pi_{1}(2015)$ in the $f_{1}(1285) \pi$ and $\omega \pi \pi$ channels $[42,43]$. However, this state is too broad to explain such an abrupt behavior and thus it is difficult to find a compelling explanation. Unfortunately, it is not possible to crosscheck this behavior with the $\eta \pi$ relative phase due to lack of data in the 1.8-2.0 GeV region. Moreover, fitting these features of the $P$ wave drives the position of the $a_{2}^{\prime}$ to unphysical values. For these reasons, we fit data up to $2 \mathrm{GeV}$ only.

Enforcing unitarity allows us to properly implement the interference among the various resonances and the background. In principle, one wishes to include all the kinematically allowed channels in a unitary analysis. Recently, COMPASS published the complete $3 \pi$ partial-wave analysis [32], including the exotic $1^{-+}$wave in the $\rho \pi$ final state. However, the extraction of the resonance pole in this channel is hindered by the irreducible Deck process [44], which refers to the exchange of a pion between the final state $\rho$ and $\pi$ (cf. Fig. 8 in Ref. [32]). This generates a peaking background in the exotic partial wave $[45,46]$. Since the Deck mechanism is not fully accounted for in the COMPASS amplitude model, we do not include the $3 \pi$ data in our analysis. As discussed in Ref. [40], neglecting additional channels does not affect the pole position, as long as the resonance poles are far away from threshold, which is the case studied here.

Reaction model.-At high energies, peripheral production of $\pi p \rightarrow \eta^{(\prime)} \pi p$ is dominated by Pomeron $(\mathbb{P})$ exchange. The notion of Pomeron exchange emerges from Regge theory [47,48], and allows us to factorize the $\pi \mathbb{P} \rightarrow$ $\eta^{(\prime)} \pi$ process. For fixed $t_{1}$ the latter resembles an ordinary helicity partial wave amplitude $a_{i}^{J}(s)$, with $i=\eta^{(\prime)} \pi$ the final channel index, $J$ the angular momentum of the $\eta^{(\prime)} \pi$ system, and $s$ its invariant mass squared. This amplitude, in principle, also depends on the effective spin and helicity of the Pomeron. However, the approximately constant hadron cross section at high energies implies that the effective spin of the Pomeron is near 1, which explains dominance of the partial wave components with angular momentum projection $M= \pm 1$ as seen in data $[33,49,50]$. Since the two are related by parity, we drop reference to the Pomeron quantum numbers (for more details, see Ref. [40]). As discussed previously, we fix an effective value $t_{\text {eff }}=-0.1 \mathrm{GeV}^{2}$.

We parametrize the amplitudes following the coupledchannel $N / D$ formalism [51],

$$
a_{i}^{J}(s)=q^{J-1} p_{i}^{J} \sum_{k} n_{k}^{J}(s)\left[D^{J}(s)^{-1}\right]_{k i},
$$

where $p_{i}$ is the $\eta^{(\prime)} \pi$ breakup momentum, and $q$ the $\pi$ beam momentum in the $\eta^{(\prime)} \pi$ rest frame. (One unit of incoming momentum $q$ is divided out because of the Pomeronnucleon vertex [40]). The $n_{k}^{J}(s)$ 's incorporate exchange "forces" in the production process and are smooth 

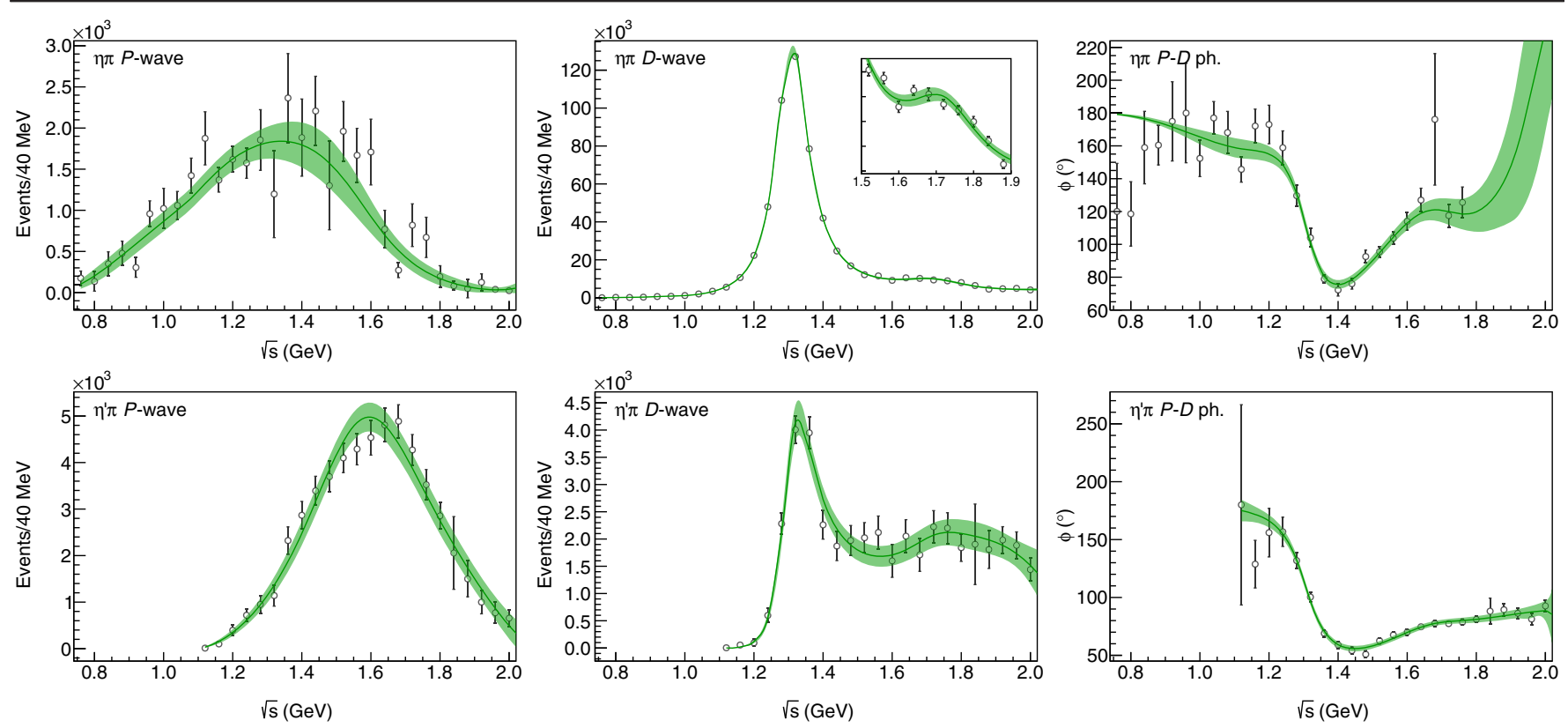

FIG. 1. Fits to the $\eta \pi$ (upper line) and $\eta^{\prime} \pi$ (lower line) data from COMPASS [33]. The intensities of the $P$ (left), $D$ wave (center), and their relative phase (right) are shown. The inset zooms into the region of the $a_{2}^{\prime}(1700)$. The solid line and green band show the result of the fit and the $2 \sigma$ confidence level provided by the bootstrap analysis, respectively. The initialization of the fit is chosen by randomly generating $O\left(10^{5}\right)$ different sets of values for the parameters. The best fit has $\chi^{2} /$ dof $=162 / 122=1.3$. The errors shown are statistical only.

functions of $s$ in the physical region. The $D^{J}(s)$ matrix represents the $\eta^{(\prime)} \pi \rightarrow \eta^{(\prime)} \pi$ final state interactions, and contains cuts on the real axis above thresholds (right-hand cuts), which are constrained by unitarity.

For the numerator $n_{k}^{J}(s)$, we use an effective expansion in Chebyshev polynomials. A customary parametrization of the denominator is given by [52]

$$
D_{k i}^{J}(s)=\left[K^{J}(s)^{-1}\right]_{k i}-\frac{s}{\pi} \int_{s_{k}}^{\infty} d s^{\prime} \frac{\rho N_{k i}^{J}\left(s^{\prime}\right)}{s^{\prime}\left(s^{\prime}-s-i \epsilon\right)},
$$

where $s_{k}$ is the threshold in channel $k$ and

$$
\rho N_{k i}^{J}\left(s^{\prime}\right)=\delta_{k i} \frac{\lambda^{J+1 / 2}\left(s^{\prime}, m_{\eta^{\prime \prime}}^{2}, m_{\pi}^{2}\right)}{\left(s^{\prime}+s_{L}\right)^{2 J+1+\alpha}}
$$

is an effective description of the left-hand singularities in the $\eta^{(\prime)} \pi \rightarrow \eta^{(\prime)} \pi$ scattering, which is controlled by the $s_{L}$ parameter fixed at the hadronic scale $\simeq 1 \mathrm{GeV}^{2}$. Finally,

$$
K_{k i}^{J}(s)=\sum_{R} \frac{g_{k}^{J, R} g_{i}^{J, R}}{m_{R}^{2}-s}+c_{k i}^{J}+d_{k i}^{J} s,
$$

with $c_{k i}^{J}=c_{i k}^{J}$ and $d_{k i}^{J}=d_{i k}^{J}$, is a standard parametrization for the $K$ matrix. In our reference model, we consider two $K$-matrix poles in the $D$ wave, and one single $K$-matrix pole in the $P$ wave; the numerator of each channel and wave is described by a third-order polynomial. We set $\alpha=2$ in
Eq. (3), which has been effective in describing the singlechannel case [40]. The remaining 37 parameters are fitted to data, by performing a $\chi^{2}$ minimization with MINUIT [53]. As shown in Fig. 1, the result of the best fit is in good agreement with data. In particular, a single $K$-matrix pole is able to correctly describe the $P$-wave peaks in the two channels, which are separated by $200 \mathrm{MeV}$. The shift of the peak in the $\eta \pi$ spectrum to lower energies originates from the combination between final state interactions and the production process. The uncertainties on the parameters are estimated via the bootstrap method [54,55]: we generate a large number of pseudo datasets and refit each one of them. The (co)variance of the parameters provides an estimate of their statistical uncertainties and correlations. The values of the fitted parameters and their covariance matrix are provided in the Supplemental Material [56]. The average curve passes the Gaussian test in Ref. [57].

Once the parameters are determined, the amplitudes can be analytically continued to complex values of $s$. The $D^{J}(s)$ matrix in Eq. (2) can be continued underneath the unitarity cut into the closest unphysical Riemann sheet. A pole $s_{P}$ in the amplitude appears when the determinant of $D^{J}\left(s_{P}\right)$ vanishes. Poles close to the real axis influence the physical region and can be identified as resonances, whereas further singularities are likely to be artifacts of the specific model with no direct physical interpretation. For any practical parametrization, especially in a coupledchannel problem, it is not possible to specify a priori the number of poles. Appearance of spurious poles far from 


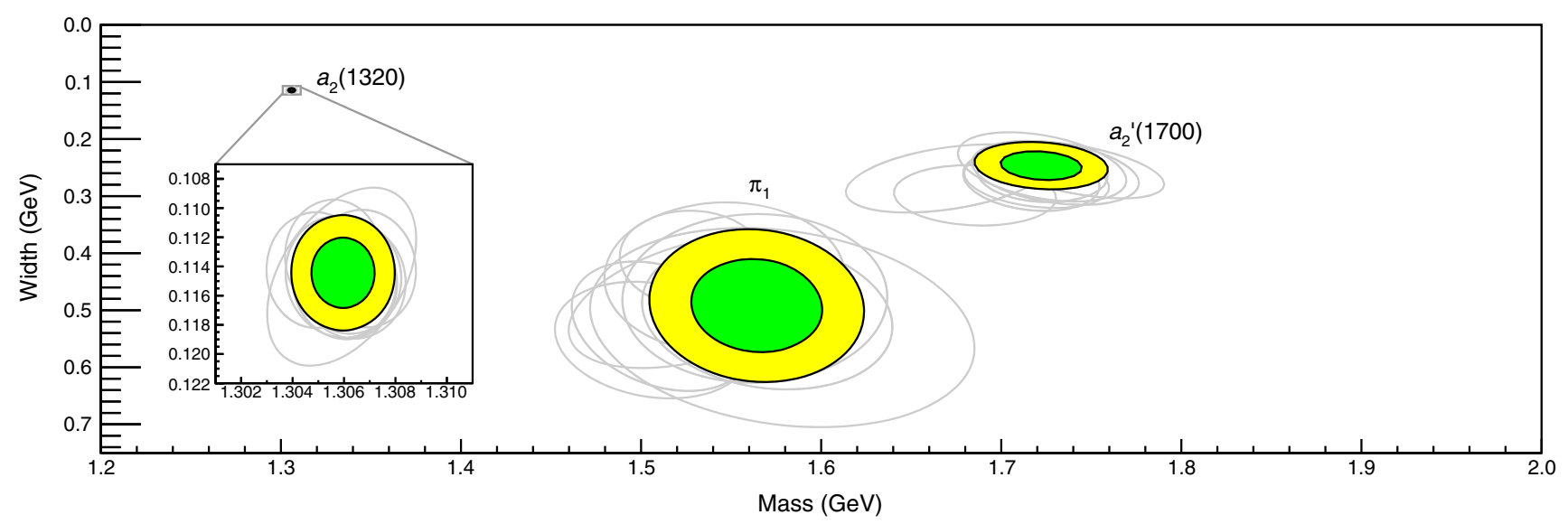

FIG. 2. Positions of the poles identified as the $a_{2}(1320), \pi_{1}$, and $a_{2}^{\prime}(1700)$. The inset shows the position of the $a_{2}(1320)$. The green and yellow ellipses show the $1 \sigma$ and $2 \sigma$ confidence levels, respectively. The gray ellipses in the background show, within $2 \sigma$, variation of the pole position upon changing the functional form and the parameters of the model, as discussed in the text.

the physical region is thus unavoidable. It is, however, possible to isolate the physical poles by testing their stability against different parametrizations and data resampling. We select the resonance poles in the $m \in[1,2]$ and $\Gamma \in[0,1] \mathrm{GeV}$ region, where customarily $m=\operatorname{Re} \sqrt{s_{P}}$ and $\Gamma=-2 \operatorname{Im} \sqrt{s_{P}}$. We find two poles in the $D$ wave, identified as the $a_{2}(1320)$ and $a_{2}^{\prime}(1700)$, and a single pole in the $P$ wave, which we call $\pi_{1}$. The pole positions are shown in Fig. 2, and the resonance parameters in Table I. To estimate the statistical significance of the $\pi_{1}$ pole, we perform fits using a pure background model for the $P$ wave, i.e., setting $g_{\eta^{(\prime)} \pi}^{P, 1}=0$ in Eq. (4). The best solution having no poles in our reference region has a $\chi^{2}$ almost 50 times larger, which rejects the possibility for the $P$-wave peaks to be generated by nonresonant production. We also considered solutions having two isolated $P$-wave poles in the reference region, which would correspond to the scenario discussed in the PDG [58]. The $\chi^{2}$ for this case is equivalent to the single pole solution. One of the poles is compatible with the previous determination, while the second is unstable; i.e., it can appear in a large region of the $s$ plane depending on the initial values of the fit parameters. Moreover, the behavior of the $\eta \pi$ phase required by the fit is rather peculiar. A $180^{\circ}$ jump (due to a zero in the amplitude) appears above $1.8 \mathrm{GeV}$, where no data exist. We conclude there is no evidence for a second pole.

TABLE I. Resonance parameters. The first error is statistical, the second systematic.

\begin{tabular}{lcc}
\hline \hline Poles & Mass $(\mathrm{MeV})$ & Width $(\mathrm{MeV})$ \\
\hline$a_{2}(1320)$ & $1306.0 \pm 0.8 \pm 1.3$ & $114.4 \pm 1.6 \pm 0.0$ \\
$a_{2}^{\prime}(1700)$ & $1722 \pm 15 \pm 67$ & $247 \pm 17 \pm 63$ \\
$\pi_{1}$ & $1564 \pm 24 \pm 86$ & $492 \pm 54 \pm 102$ \\
\hline \hline
\end{tabular}

Systematic uncertainties.-Unlike the COMPASS mass independent fit, the pole extraction carries systematic uncertainties associated with the reaction model. To assess these, we vary the parameters and functional forms which were kept fixed in the previous fits. We can separate these in two categories: (i) variations of the numerator function $n_{k}^{J}(s)$ in Eq. (1), which is expected to be smooth in the region of the data, and (ii) variations of the function $\rho N\left(s^{\prime}\right)$, which determines the imaginary part of the denominator in Eq. (2). As for the latter, we investigate whether the specific form we chose biases the determination of the poles. Upon variation of the parameters and of the functional forms, the shape of the dispersive integral in Eq. (2) is altered, but the fit quality is unaffected. The pole positions change roughly within $2 \sigma$, as one can see in Fig. 2. As for the numerator $n_{k}^{J}(s)$, we varied the effective value of $t_{\text {eff }}$ and the order of the polynomial expansion. Given the flexibility of the numerator parametrization, these variations effectively absorb the model dependence related to the production mechanism. None of these cause important changes in pole locations. Our final estimate for the uncertainties is reported in Table I, while the detailed summary is given in the Supplemental Material [56].

Conclusions. -We performed the first coupled-channel analysis of the $P$ and $D$ waves in the $\eta^{(\prime)} \pi$ system measured at COMPASS [33]. We used an amplitude parametrization constrained by unitarity and analyticity. We find two poles in the $D$ wave, which we identify as the $a_{2}(1320)$ and the $a_{2}^{\prime}(1700)$, with resonance parameters consistent with the single-channel analysis [40]. In the $P$ wave, we find a single exotic $\pi_{1}$ in the region constrained by data. This determination is compatible with the existence of a single isovector hybrid meson with quantum numbers $J^{P C}=1^{-+}$, as suggested by lattice QCD [13-15]. Its mass and width are determined to be $1564 \pm 24 \pm 86$ and $492 \pm 54 \pm 102 \mathrm{MeV}$, respectively. The statistical uncertainties are estimated via the bootstrap technique, while the 
systematics due to model dependence are assessed by varying parameters and functional forms that are not directly constrained by unitarity. We find no evidence for a second pole that could be identified with another $\pi_{1}$ resonance. Solutions with two poles are possible, but do not improve the fit quality and, when present, the position of the second pole is unstable against different starting values of the fit. It is worth noting that the two-pole solutions have a peculiar behavior of the $\eta \pi$ phase in the $\gtrsim 2 \mathrm{GeV}$ mass region, where no data exist. New data from GlueX and CLAS12 experiments at Jefferson Lab in this and higher mass region will be valuable to test this behavior.

We would like to thank the COMPASS Collaboration for useful comments. A.P. thanks the Mainz Institute for Theoretical Physics (MITP) for its kind hospitality while this work was being completed. This work was supported by the U.S. Department of Energy under Grants No. DE-AC0506OR23177 and No. DE-FG02-87ER40365, U.S. National Science Foundation under Grant No. PHY-1415459, Ministerio de Ciencia, Innovación y Universidades (Spain) Grants No. FPA2016-75654-C2-2-P and No. FPA201677313-P, Universidad Complutense de Madrid predoctoral scholarship program, Research Foundation-Flanders (FWO), PAPIIT-DGAPA (UNAM, Mexico) under Grants No. IA101717 and No. IA101819, CONACYT (Mexico) under Grant No. 251817, the German Bundesministerium für Bildung und Forschung (BMBF), and Deutsche Forschungsgemeinschaft (DFG) through the Collaborative Research Center [The Low-Energy Frontier of the Standard Model (SFB 1044)] and the Cluster of Excellence [Precision Physics, Fundamental Interactions and Structure of Matter (PRISMA)].

*arodas@ucm.es pillaus@jlab.org

[1] B. Ketzer, Proc. Sci., QNP2012 (2012) 025.

[2] C. A. Meyer and E. S. Swanson, Prog. Part. Nucl. Phys. 82, 21 (2015).

[3] A. Esposito, A. Pilloni, and A. D. Polosa, Phys. Rep. 668, 1 (2017).

[4] R. F. Lebed, R. E. Mitchell, and E. S. Swanson, Prog. Part. Nucl. Phys. 93, 143 (2017).

[5] F.-K. Guo, C. Hanhart, U.-G. Meißner, Q. Wang, Q. Zhao, and B.-S. Zou, Rev. Mod. Phys. 90, 015004 (2018).

[6] S. L. Olsen, T. Skwarnicki, and D. Zieminska, Rev. Mod. Phys. 90, 015003 (2018).

[7] M. Karliner, J. L. Rosner, and T. Skwarnicki, Annu. Rev. Nucl. Part. Sci. 68, 17 (2018).

[8] D. Horn and J. Mandula, Phys. Rev. D 17, 898 (1978).

[9] N. Isgur and J. E. Paton, Phys. Rev. D 31, 2910 (1985).

[10] M. S. Chanowitz and S. R. Sharpe, Nucl. Phys. B222, 211 (1983); B228, 588 (1983).

[11] T. Barnes, F. Close, F. de Viron, and J. Weyers, Nucl. Phys. B224, 241 (1983).

[12] F. E. Close and P. R. Page, Nucl. Phys. B443, 233 (1995).
[13] P. Lacock, C. Michael, P. Boyle, and P. Rowland (UKQCD Collaboration), Phys. Lett. B 401, 308 (1997).

[14] C. Bernard, J. E. Hetrick, T. A. DeGrand, M. Wingate, C. DeTar, C. McNeile, S. Gottlieb, U. M. Heller, K. Rummukainen, B. Sugar, and D. Toussaint (MILC Collaboration), Phys. Rev. D 56, 7039 (1997).

[15] J. J. Dudek, R. G. Edwards, P. Guo, and C. E. Thomas (Hadron Spectrum Collaboration), Phys. Rev. D 88, 094505 (2013).

[16] A. P. Szczepaniak and E. S. Swanson, Phys. Rev. D 65, 025012 (2001).

[17] A. P. Szczepaniak and P. Krupinski, Phys. Rev. D 73, 116002 (2006).

[18] P. Guo, A. P. Szczepaniak, G. Galata, A. Vassallo, and E. Santopinto, Phys. Rev. D 78, 056003 (2008).

[19] S. D. Bass and P. Moskal, arXiv:1810.12290.

[20] G. Baum et al. (COMPASS Collaboration), http://cds.cern .ch/record/298433 (1996).

[21] P. Abbon et al. (COMPASS Collaboration), Nucl. Instrum. Methods Phys. Res., Sect. A 779, 69 (2015).

[22] A. Rizzo (CLAS Collaboration), J. Phys. Conf. Ser. 689, 012022 (2016).

[23] S. Dobbs (GlueX Collaboration), Proc. Sci., Hadron2017 (2018) 047.

[24] D. R. Thompson et al. (E852 Collaboration), Phys. Rev. Lett. 79, 1630 (1997).

[25] S. U. Chung et al. (E852 Collaboration), Phys. Rev. D 60, 092001 (1999).

[26] G. S. Adams et al. (E852 Collaboration), Phys. Lett. B 657, 27 (2007).

[27] A. Abele et al. (Crystal Barrel Collaboration), Phys. Lett. B 423, 175 (1998).

[28] A. Abele et al. (Crystal Barrel Collaboration), Phys. Lett. B 446, 349 (1999).

[29] E. I. Ivanov et al. (E852 Collaboration), Phys. Rev. Lett. 86, 3977 (2001).

[30] Yu. A. Khokhlov (VES Collaboration), Nucl. Phys. A663A664, 596c (2000); Particles and nuclei, in Proceedings of the 15th International Conference, PANIC '99, Uppsala, Sweden, 1999.

[31] M. Alekseev et al. (COMPASS Collaboration), Phys. Rev. Lett. 104, 241803 (2010).

[32] R. Akhunzyanov et al. (COMPASS Collaboration), Phys. Rev. D 98, 092003 (2018).

[33] C. Adolph et al. (COMPASS Collaboration), Phys. Lett. B 740, 303 (2015).

[34] S. D. Bass and E. Marco, Phys. Rev. D 65, 057503 (2002).

[35] A. P. Szczepaniak, M. Swat, A. R. Dzierba, and S. Teige, Phys. Rev. Lett. 91, 092002 (2003).

[36] F. E. Close and H. J. Lipkin, Phys. Lett. B 196, 245 (1987).

[37] S. U. Chung, E. Klempt, and J. G. Korner, Eur. Phys. J. A 15, 539 (2002).

[38] R. L. Jaffe and F. Wilczek, Phys. Rev. Lett. 91, 232003 (2003).

[39] R. Jaffe, Phys. Rep. 409, 1 (2005).

[40] A. Jackura et al. (COMPASS and JPAC Collaborations), Phys. Lett. B 779, 464 (2018).

[41] T. Schlüter, The $\pi^{-} \eta$ and $\pi^{-} \eta^{\prime}$ systems in exclusive $190 \mathrm{GeV}$ $\pi^{-} p$ reactions at COMPASS, Ph.D. thesis, Munich University, 2012. 
[42] J. Kuhn et al. (E852 Collaboration), Phys. Lett. B 595, 109 (2004).

[43] M. Lu et al. (E852 Collaboration), Phys. Rev. Lett. 94, 032002 (2005).

[44] R. T. Deck, Phys. Rev. Lett. 13, 169 (1964).

[45] G. Ascoli, R. Cutler, L. M. Jones, U. Kruse, T. Roberts, B. Weinstein, and H. W. Wyld, Phys. Rev. D 9, 1963 (1974).

[46] D. Ryabchikov, Analysis of $\pi^{-} \pi^{-} \pi^{+}$in COMPASS data: role of $a_{1}(1260)$ meson and Deck process, https://indico .cern.ch/event/591374/contributions/2498368/attachments/ 1427728/2191292/03_ryabchikov_athos2017.pdf.

[47] G. F. Chew and S. C. Frautschi, Phys. Rev. Lett. 7, 394 (1961).

[48] S. Donnachie, H. G. Dosch, O. Nachtmann, and P. Landshoff, Pomeron Physics and QCD, Cambridge Monographs on Particle Physics, Nuclear Physics and Cosmology (Cambridge University Press, Cambridge, 2005).

[49] F. E. Close and G. A. Schuler, Phys. Lett. B 458, 127 (1999).
[50] T. Arens, O. Nachtmann, M. Diehl, and P. V. Landshoff, Z. Phys. C 74, 651 (1997).

[51] J. D. Bjorken, Phys. Rev. Lett. 4, 473 (1960).

[52] I. J. R. Aitchison, Nucl. Phys. A189, 417 (1972).

[53] F. James and M. Roos, Comput. Phys. Commun. 10, 343 (1975).

[54] W. H. Press, S. A. Teukolsky, W. T. Vetterling, and B. P. Flannery, Numerical Recipes: The Art of Scientific Computing, 3rd ed. (Cambridge University Press, New York, NY, USA, 2007).

[55] B. Efron and R. Tibshirani, An Introduction to the Bootstrap, Chapman \& Hall/CRC Monographs on Statistics \& Applied Probability (CRC Press, Baco Raton, 1994).

[56] See Supplemental Material at http://link.aps.org/ supplemental/10.1103/PhysRevLett.122.042002 for additional information about fit results and systematic studies.

[57] R. Navarro Pérez, E. Ruiz Arriola, and J. Ruiz de Elvira, Phys. Rev. D 91, 074014 (2015).

[58] M. Tanabashi et al. (Particle Data Group), Phys. Rev. D 98, 030001 (2018). 